\title{
Microbial diversity in the saline-alkali soil of a coastal Tamarix chinensis woodland at Bohai Bay, China
}

\author{
LIU Wanqiu ${ }^{1,2,3}$, ZHANG Wei ${ }^{1,2}$, LIU Guangxiu ${ }^{1,2^{*}}$, ZHANG Yanhua ${ }^{3}$, ZHANG Gaosen ${ }^{1,2}$ \\ ${ }^{1}$ Key Laboratory of Desert and Desertification, Cold and Arid Regions Environmental and Engineering Research Institute, \\ Chinese Academy of Sciences, Lanzhou 730000, China; \\ ${ }^{2}$ Key Laboratory of Extreme Environmental Microbial Resources and Engineering, Gansu Province, Lanzhou 730000, China; \\ ${ }^{3}$ Ocean and Fishery Bureau of Changyi, Changyi 261300, China
}

\begin{abstract}
Soil salinization or alkalization is a form of soil desertification. Coastal saline-alkali soil represents a type of desert and a key system in the network of ecosystems at the continent-ocean interface. Tamarix chinensis is a drought-tolerant plant that is widely distributed in the coastal saline-alkali soil of Bohai Bay, China. In this study, we used 454 pyrosequencing techniques to investigate the characteristics and distribution of the microbial diversity in coastal saline-alkali soil of the T. chinensis woodland at Bohai Bay. A total of 20,315 sequences were obtained, representing 19 known bacterial phyla and a large proportion of unclassified bacteria at the phylum level. Proteobacteria, Acidobacteria and Actinobacteria were the predominant phyla. The coverage of $T$. chinensis affected the microbial composition. At the phylum level, the relative abundance of y-Proteobacteria and Bacteroidetes decreased whereas Actinobacteria increased with the increasing coverage of $T$. chinensis. At the genus level, the proportions of Steroidobacter, Lechevalieria, Gp3 and Gp4 decreased with the increase of the vegetation coverage whereas the proportion of Nocardioides increased. A cluster analysis showed that the existing $T$. chinensis changed the niches for the microorganisms in the coastal saline-alkali soil, which caused changes in the microbial community. The analysis also distinguished the microbial community structure of the marginal area from those of the dense area and sparse area. Furthermore, the results also indicated that the distance to the seashore line could also affect certain groups of soil bacteria in this coastal saline-alkali soil, such as the family Cryomorphaceae and class Flavobacteria, whose population decreased as the distance increased. In addition, the seawater and temperature could be the driving factors that affected the changes.
\end{abstract}

Keywords: coastal saline-alkali soil; Tamarix chinensis; bacteria; pyrosequencing

Citation: LIU Wanqiu, ZHANG Wei, LIU Guangxiu, ZHANG Yanhua, ZHANG Gaosen. 2016. Microbial diversity in the saline-alkali soil of a coastal Tamarix chinensis woodland at Bohai Bay, China. Journal of Arid Land, 8(2): 284-292. doi: $10.1007 / \mathrm{s} 40333-015-0092-2$

Soil salinization or alkalization is a form of soil desertification. Coastal saline-alkali soil is a common form of soil salinization and alkalization, which represents a type of desert (Szabolcs, 1992; Thomas and Middleton, 1993). This typical arid land represents a key ecosystem at the continent-ocean interface (Carpentier et al., 2013) and has high continental and marine nutrient inputs due to a high microbial community turnover and high mineralization of organic matter. However, this ecosystem is ecologically and economically vulnerable (Zhu et al., 2012). It may be exposed to a variety of organic pollutants from oil and chemical spills (Wilms et al., 2006a, b;

*Corresponding author: LIU Guangxiu (E-mail: liugx@1zb.ac.cn)
Received 2015-05-11; revised 2015-10-22; accepted 2015-10-26
C Xinjiang Institute of Ecology and Geography, Chinese Academy of Sciences, Science Press and Springer-Verlag Berlin Heidelberg 2016 
Kim et al., 2014). Coastal saline-alkali soil has high primary production rate and, in turn, intense heterotrophic activity (Wilms et al., 2006a). Previous studies suggested that such high microbial activity led to oxygen depletion within the first few millimeters of the sediments, below the prevailing anoxic condition (Graue et al., 2011). The microbial diversity of coastal saline-alkali soil was analyzed using culture-independent or cultivation-based methods. Proteobacteria and Bacteroidetes were the dominant phyla found from the surface to $20 \mathrm{~cm}$ depth in the sediment (Llobet-Brossa et al., 1998), and other studies showed that only Proteobacteria could be the dominant phylum in coastal saline-alkali soil (Kim et al., 2004; Köpke et al., 2005).

Tamarix is an Old World genus that has grown in the Mediterranean region since the Tertiary Period. The Tamarix genus is widely distributed in eastern and southern Asia, southern Europe, the Mediterranean, the Middle East and North Africa. The genus includes typical desert plants and contains approximately 54 species of shrubs and trees. Most of these species occur in salty, dry, or riparian habitats (Gaskin and Schaal, 2002). They adapted to saline soils by excreting the excessive salts out through foliar glands. As a consequence, they can promote soil de-salinization in their vicinity (Cao et al., 2011). Tamarix chinensis is native to China, Mongolia and Japan and is one of the dominant salt-tolerant plant species in the coastal saline-alkali soil of Bohai Bay, China (Gaskin and Schaal, 2002; Hou et al., 2012; Zhu et al., 2012). As an early riparian species with specific adaptations to flood and salt conditions, T. chinensis is a perennial shrub that grows along the coastal shores in the Yellow River Delta. This plant plays an important role in preventing soil erosion, regulating the microclimate and retaining coastal saline-alkali soil ecological stability in the region (Jiang et al., 2012).

To date, limited researches have been conducted on the microorganisms related to Tamarix. Phyllosphere bacterial populations were investigated and the results indicated that the geographical distance was the most important driving factor for the microbial community in the Tamarix phyllosphere (Finkel et al., 2012; Qvit-Raz et al., 2012). The differences in the microbial communities in the rhizosphere and non-rhizosphere of Tamarix were also investigated (Ma and Yin, 2009). Moreover, several studies showed that the extracts of Tamarix had antimicrobial activities (Sultanova et al., 2001; Saïdana et al., 2008; Ksouri et al., 2009; Lefahal et al., 2010). Regarding the soil microbial community in Tamarix woodlands, only one study surveyed the seasonal changes of the soil microbial communities in a T. chinensis community from the Yellow River Delta using phospholipid fatty acid (PLFA) methods (Cao et al., 2011). Therefore, it was interesting to investigate the microbial composition in coastal saline-alkali soil under the Tamarix woodland and the influence of the plants on the microorganisms in this ecosystem.

In this study, we attempted to investigate the characteristics and distribution of soil microbial diversity in the $T$. chinensis woodland in the coastal saline-alkali soil of Bohai Bay. The results, with a much higher resolution than that of PLFA, could provide the first insight into the microbial community in coastal saline-alkali soil of this study area and show the influence of tree cover on the soil microbial composition.

\section{Materials and methods}

\subsection{Study area and sampling}

Soil samples were mainly collected in November 2012 and the sampling site $\left(37^{\circ} 05^{\prime} \mathrm{N}, 119^{\circ} 21^{\prime} \mathrm{E}\right)$ is located at Bohai Bay, China. Three sites were selected to represent dense, marginal and sparse tree coverages, with the coverages defined as high $(>80 \%)$, intermediate $(60 \%-80 \%)$ and low $(<10 \%)$, respectively. Five soil cores in 5 -cm diameter were randomly collected from each coverage type, and the upper $10 \mathrm{~cm}$ of the five cores were mixed and pooled in a gas-tight bottle. The else soil samples were collected in September 2012 from high vegetation coverage sites to assess seasonal variations. Samples were transported in an icebox and stored at $-80^{\circ} \mathrm{C}$ in the laboratory before further analysis. 


\subsection{DNA extraction, Polymerase Chain Reaction (PCR) amplification and pyrosequencing}

Before DNA extraction, the samples were sieved through a 2-mm mesh for thorough homogenization and to remove roots and plant detritus (Dong et al., 2013; Dhar et al., 2015). Genomic DNA was isolated from 1 to $1.25 \mathrm{~g}$ of mixed soil using the PowerSoil DNA Isolation Kit (MoBio Laboratories, Carlsbad, CA). The extracted DNA was checked by $1 \%$ agarose gel, and the concentration was determined using a Nanodrop ND-1000 UV-Vis Spectrophotometer (Nano-Drop Technologies, Wilmington, DE). The eluted DNA was stored at $-20^{\circ} \mathrm{C}$.

The 16S rRNA gene fragments in appropriate size and sequence variability were amplified with primer 341F (CCTAYGGGRBGCASCAG) and a modified primer 806R (GGACTACNNGGG TATCTAAT) (Yu et al., 2005). By using these primers, the V1-V3 region of the $16 \mathrm{~S}$ rRNA gene was amplified. Each PCR reaction contained $2 \mu \mathrm{L} 10 \times$ buffer, $1.6 \mu \mathrm{L}$ dNTP, $0.8 \mu \mathrm{L}$ forward primer, $0.8 \mu \mathrm{L}$ reverse primer and $0.8 \mu \mathrm{L} 1 \mathrm{Pfu}$ Taq DNA polymerase. Thermal cycling consisted of an initial denaturation at $94^{\circ} \mathrm{C}$ for $3 \mathrm{~min}$, followed by 25 cycles of denaturation at $94^{\circ} \mathrm{C}$ for $30 \mathrm{~s}$, annealing at $50^{\circ} \mathrm{C}$ for $30 \mathrm{~s}$, an extension at $72^{\circ} \mathrm{C}$ for $30 \mathrm{~s}$ and a final extension of $10 \mathrm{~min}$ at $72^{\circ} \mathrm{C}$.

Amplicons were purified using the AxyPrep DNA Gel Extraction Kit (Axygen). Concentrations of the amplicons were quantified by a TBS-380 system, and equal amount of the amplicons were mixed in a single tube. The emulsion PCR was carried out using techniques noted by Margulies et al. (2005), and sequencing was performed on a GS FLX Titanium System (454 Life Sciences, Roche Applied Science).

\subsection{Phylogenetic assignment, alignment and clustering of 16S rRNA gene fragments}

All reads were processed with mothur, according to the procedure described by Schloss et al. (2011). Sequences were depleted of barcodes and primers and then subjected to quality control. Short sequences with less than 200 base pairs and sequences with an ambiguous base or with homopolymer runs exceeding $6 \mathrm{bp}$ were removed. Sequences were then denoised and the chimeras were removed (Roesch et al., 2007; McKenna et al., 2008). The remaining high quality reads were assigned to a reference taxonomy, the SILVA reference database (Pruesse et al., 2007), using the RDP naïve Bayesian rRNA classifier (confidence threshold, 80\%). Operational taxonomic units (OTUs) were defined after the removal of singleton sequences, clustering at 3\% divergence ( $97 \%$ similarity). This classification was used for all further downstream analyses on all taxonomic levels. Phylotypes were identified using Megablast and the representative sequence from each phylotype was aligned using the Ribosomal Database Project (RDP) (Cole et al., 2007).

\subsection{Statistical analysis}

Diversity and richness estimators were made at the $3 \%$ dissimilarity level with DOTUR (Roesch et al. 2007). A cluster analysis was performed using a hierarchical cluster analysis from the SPSS version 16 (SPSS, Inc., Chicago, IL).

\section{Results}

A total of 20,315 valid sequences were obtained through pyrosequencing assays (Table 1). A total

Table 1 Vegetation coverage, pyrosequencing reads, diversity and richness estimators of the three sites

\begin{tabular}{ccccccc}
\hline $\begin{array}{c}\text { Sample } \\
\text { ID }\end{array}$ & $\begin{array}{c}\text { Vegetation coverage } \\
(\%)\end{array}$ & Reads & $\begin{array}{c}\text { Chao1 } \\
\text { index }\end{array}$ & $\begin{array}{c}\text { Observed } \\
\text { Species index }\end{array}$ & $\begin{array}{c}\text { Shannon } \\
\text { index }\end{array}$ & $\begin{array}{c}\text { Simpson } \\
\text { index }\end{array}$ \\
\hline MA & 5 & 7,753 & 1,694 & 692.4 & 8.6665 & 0.9946 \\
SA & 72 & 2,260 & 1,141 & 570.0 & 8.3687 & 0.9944 \\
DA-N & 100 & 1,639 & 1,475 & 626.8 & 8.5141 & 0.9948 \\
DA-S & 100 & 8,663 & 1,607 & 693.6 & 8.7940 & 0.9962 \\
\hline
\end{tabular}

Note: MA and SA indicate the marginal area and sparse area, respectively. DA-N and DA-S indicate the soil samples collected in November and September at the dense area, respectively. 
of 1,639 sequences of each sample were selected to calculate those estimators based on the pyrosequencing reads numbers. The results indicated that the sample from SA (sparse area) had the lowest values in Chaol and observed species index.

A total of 19 phyla and a large proportion of unclassified bacteria at the phylum level were detected in all samples (Fig. 1). Proteobacteria, Acidobacteria, and Actinobacteria were abundant $(>15 \%)$. There were low proportions of some phyla and candidate divisions. The dominant bacterial phyla were similar in all samples, regardless the $T$. chinensis coverage level. The dominant soil bacterial phylum was Proteobacteria, with an abundance higher than $27.8 \%$. The phylum was subdivided into $\alpha-, \beta-, \delta$-, and $\gamma$-Proteobacteria classes. Within these classes, the $\alpha-$, $\beta$ - and $\delta$-Proteobacteria had similar proportions in the four samples, whereas the $\gamma$-Proteobacteria decreased in abundance as the vegetation coverage increased. The second most abundant phylum was Acidobacteria, with the highest abundance in the marginal area. The third most abundant phylum was Actinobacteria and its abundance increased with the increase of vegetation coverage. Bacteroidetes was the fourth most abundant phylum with the abundance decreased with the increase T. chinensis coverage. The other bacterial phyla also varied with the coverage level of $T$. chinensis (Fig. 1b). There were also several phyla found in specific areas. For example, the phyla Fibrobacteres and Tenericutes were only detected in the marginal area, and the candidate phylum OD1 was only detected in the dense area in November.

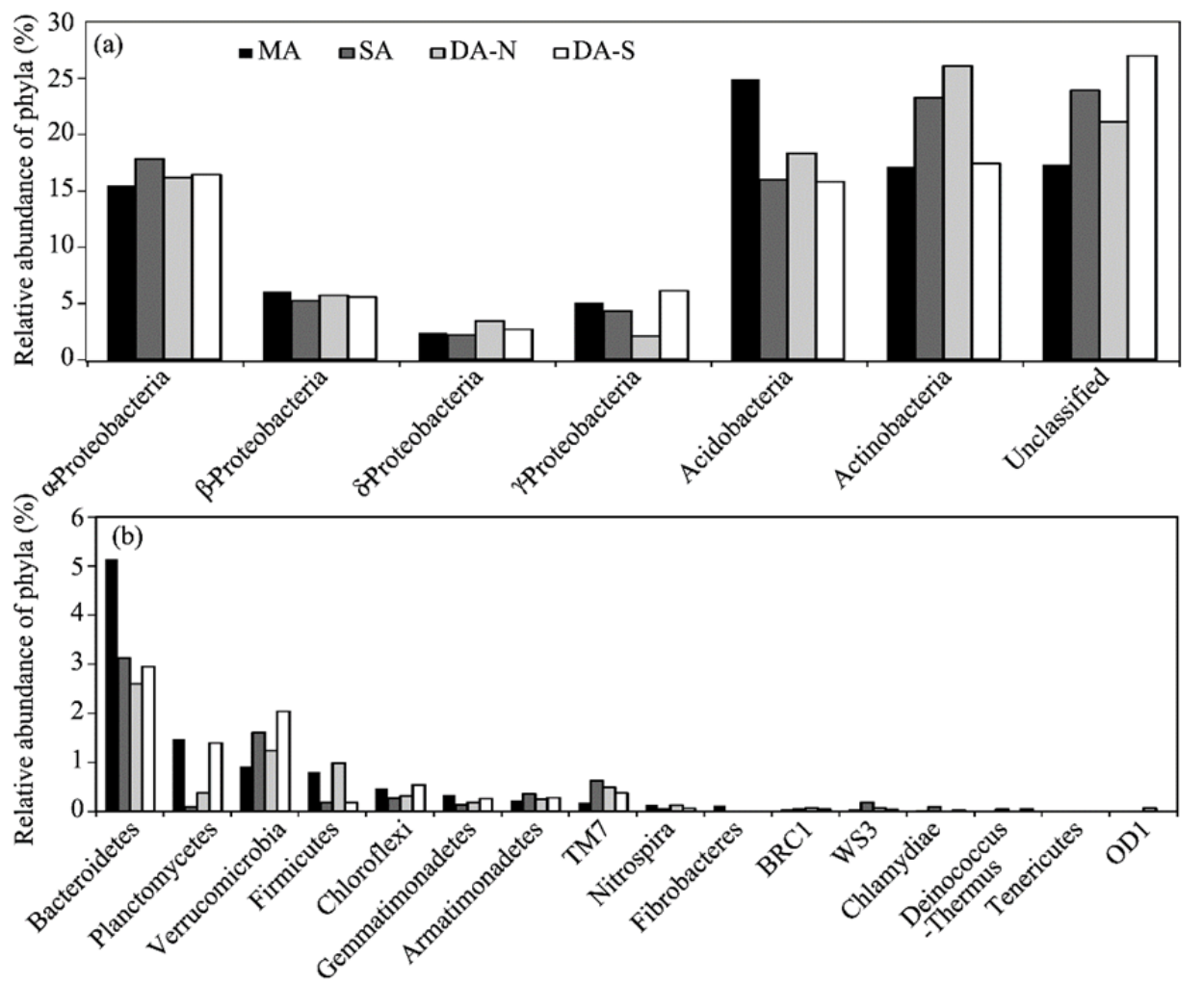

Fig. 1 Relative abundance of phyla and proteobacterial classes for each soil library

The number of genera was too large to present in one figure; therefore, only the genera with a relative abundance higher than $1 \%$ are shown in Fig. 2 . The predominant classified genera were Gp4 and Gp6, which belonged to Acidobacteria and their relative abundances reached up to $15.3 \%$ and $7.8 \%$, respectively. Some changes in the proportion of the classified genera also corresponded to the change in T. chinensis coverage within the same sampling date. For example, the proportion of Gp4, Steroidobacter, Lechevalieria and Gp3 decreased, whereas Nocardioides increased with the increase of the tree coverage. 


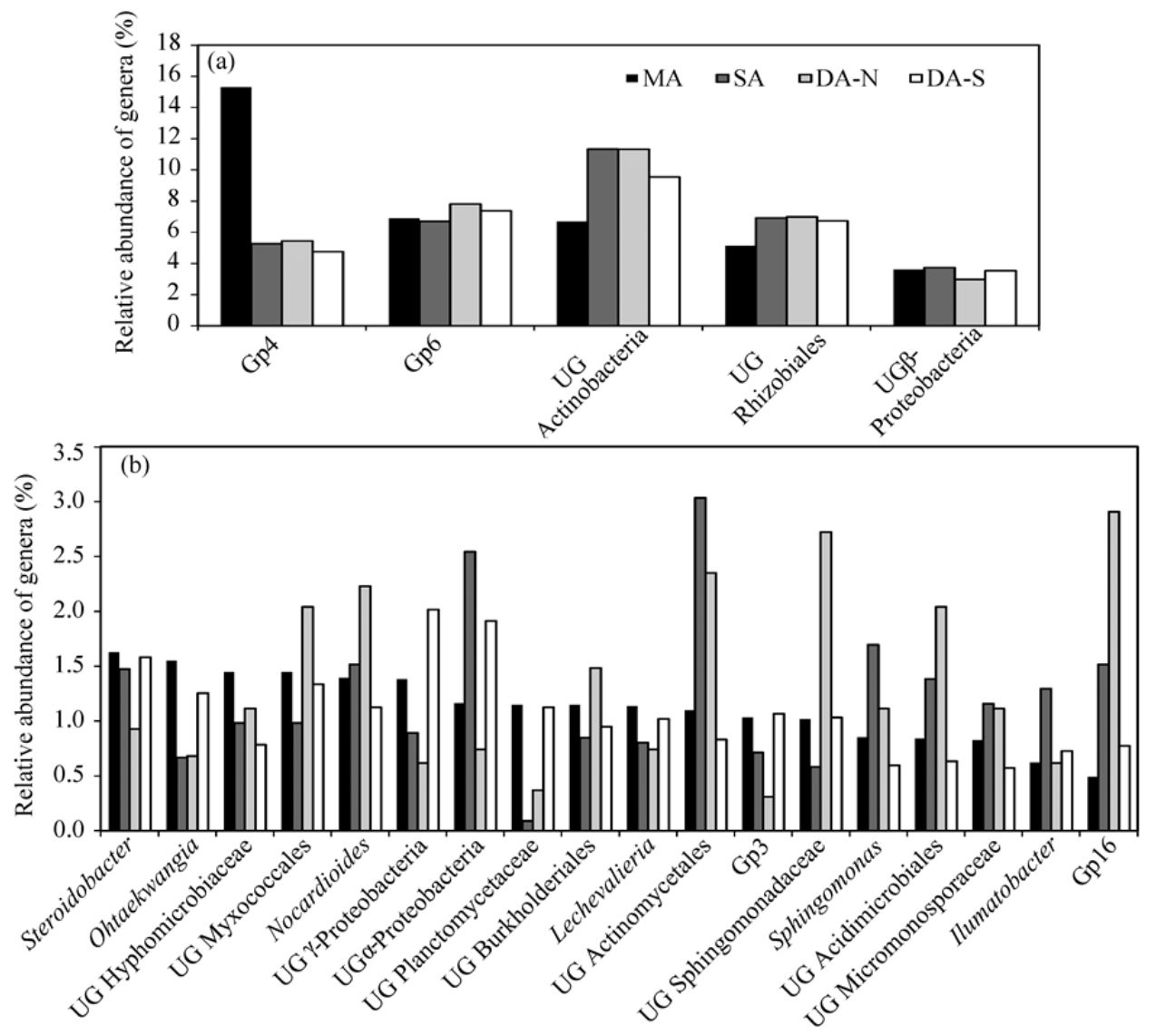

Fig. 2 Relative abundance of genera for each soil library. Only genera with more than $1 \%$ relative abundance are shown here. UG indicates an unclassified genus.

The results of the cluster analysis indicated that the vegetation coverage of $T$. chinensis had a strong effect on the bacterial composition (Fig. 3). The relative abundances at both the phylum and genus levels showed that the marginal area was different from the other sample sites. At the phylum level, the sparse and dense areas from the same sampling season were clustered together. However, at the genus level, the sparse and dense areas from different sampling season were clustered into the same groups.

Dendrogram using average linkage (between groups) rescaled distance cluster combine

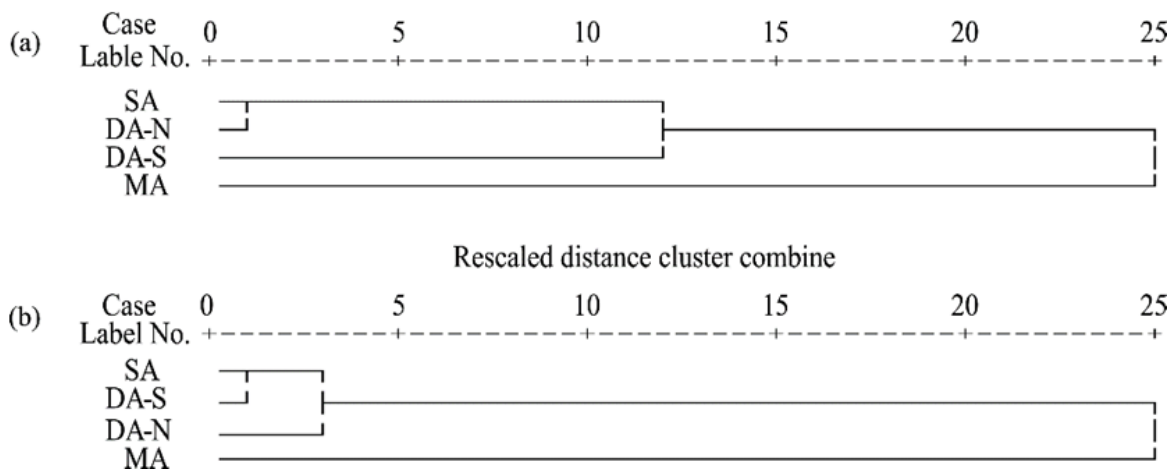

Fig. 3 Cluster analysis based on the relative abundances of (a) known phyla and (b) genera of known phyla 
The functional community also shifted along with environmental change in this coastal saline-alkali soil. The results showed that the relative abundance of the family Cryomorphaceae and class Flavobacteria decreased with the increase of T. chinensis coverage (Fig. 4).

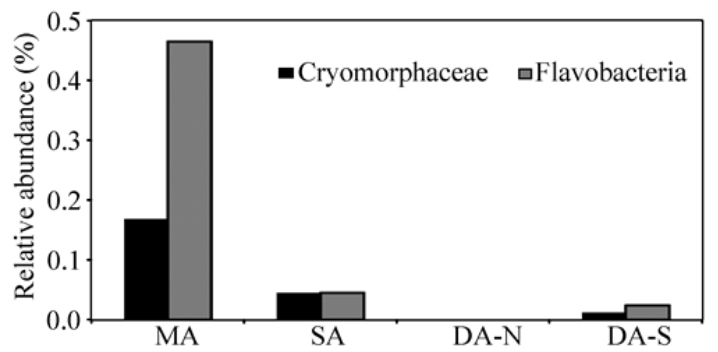

Fig. 4 Relative abundance of the family Cryomorphaceae and class Flavobacteria

\section{Discussion}

In this study, 454 pyrosequencing techniques were used to investigate the characteristics of soil microbial diversity in coastal saline-alkali soil under the T. chinensis woodland at Bohai Bay. Proteobacteria, Acidobacteria, and Actinobacteria were the dominant phyla. The relative abundance of Proteobacteria was stable in the different samples, which may be due to the arid environmental condition. The dominant phyla were widely distributed in the arid environment. Previous studies showed that Proteobacteria was always the dominant bacterial group in desert ecosystems and was ubiquitously distributed in deserts worldwide (Nagy et al., 2005; Chanal et al., 2006; Orlando et al., 2010; Saul-Tcherkas and Steinberger, 2011; Zhang et al., 2012). Meanwhile, studies using pyrosequencing and clone library methods showed that Proteobacteria could be the dominant phylum in coastal saline-alkali soil environments (Gray and Herwig, 1996; Urakawa et al., 1999; Kim et al., 2004, 2008). However, the dominant class of this phylum varied across locations (Bull et al., 2005). In addition to Proteobacteria, the predominant phyla Acidobacteria and Actinobacteria in this study were not predominant phyla in other coastal saline-alkali soil environments (Gray and Herwig, 1996; Urakawa et al., 1999; Kim et al., 2004; Kim et al., 2008). Members of these two phyla are commonly found in most types of soils. Actinobacteria was also widely distributed in desert ecosystems (Nagy et al., 2005; Chanal et al., 2006; Orlando et al., 2010; Saul-Tcherkas and Steinberger, 2011; Zhang et al., 2012). The abundance of this phylum always constituted a large percentage of the inhabitants of arid soils because it was highly resilient to low levels of soil moisture (Doroshenko et al., 2005). Acidobacteria represented, on average, $20 \%$ of the soil bacteria and was highly diverse and physiologically active in situ. Previous reports indicated members from this phylum had the ability to degrade a broad array of simple carbon compounds as well as plant and microbial polysaccharides including cellulose. This phylum was also well distributed in arid environments, especially in desert soils (Kuske et al., 2002). However, their individual functions and interactions with other higher taxa in soils are still unknown (Naether et al., 2012). In our study, the dominant classified genera were Gp4 and Gp6, which also belong to Acidobacteria. Although these genera were widely distributed in the soil, they were not well described yet (Zhang et al., 2011; Naether et al., 2012).

In addition to the dominant phyla, the Bacteroidetes, Planctomycetes, Verrucomicrobia and Firmicutes were also widely distributed in each sample. The relative abundances of these phyla were greater than $1 \%$. Rare phyla, such as Chloroflexi, Gemmatimonadetes, Armatimonadetes and TM7, had abundances less than $1 \%$ and were also detected in all of the samples. However, no significant variation trends were found. Bacterial communities are known for their large contributions to several soil ecosystem functions, such as their key role in soil sustainability (Uroz et al., 2010). Our results provide the first insight into the characteristics of microbial diversity in coastal saline-alkali soil of the study area. 
Plants living in coastal saline-alkali soil also affected the underground microbial composition. The cluster analysis results showed a tight relationship between bacterial community composition and T. chinensis coverage in this coastal saline-alkali soil. Previous studies showed that soil under the Tamarix woodland contained similar quantities of microorganisms in both coastal saline-alkali soil and the inland arid region, which indicated that the colonization of Tamarix provided stable growth conditions for microorganisms (Chen et al., 2008; Ma et al., 2008; Ma and Yin, 2009; Zhao et al., 2009). The microbial activity in coastal saline-alkali soil was higher than that of inland arid regions (Zhu et al., 2008; Zhao et al., 2009). There were no previous investigations regarding the effects of Tamarix on the underground microbial community composition. Our study provides the first insight into the relationship between the soil bacterial community composition and $T$. chinensis. The results showed that the relative abundance of $\gamma$-Proteobacteria and Bacteroidetes decreased with the increase of $T$. chinensis coverage, whereas the Actinobacteria increased with the increase of the coverage. The Fibrobacteres and Tenericutes were only detected in the marginal area, whereas OD1 was only detected in the dense area in November. A variation in the bacterial community also occurred at the genus level. The proportion of Gp4, Steroidobacter, Lechevalieria, and Gp3 decreased with the increasing in tree coverage, whereas Nocardioides increased with the increasing in tree cover. During the development of the $T$. chinensis woodland, the increase of some genera are highly likely linked to the abundance of $T$. chinensis.

The cluster analysis also revealed the effects of tree coverage on the soil bacteria composition. The marginal area was different from the other sites based on the relative abundance at the phylum or genus levels. The sparse and dense areas were close to each other, suggesting that the existence of $T$. chinensis changed the niches for the microorganisms in coastal saline-alkali soil and then distinguished the microbial community structure of the marginal area from that of the dense area and sparse area.

The results also indicated that the distance to the seashore line could also affect certain groups of soil bacteria in this coastal saline-alkali soil. For example, the relative abundance of the class Flavobacteria decreased with increasing distance from the seashore line, namely from the dense area to the sparse area, and then to the marginal area. Flavobacteria were the major decomposers of high-molecular-mass organic matter in sea water (Cottrell and Kirchman, 2000; Woyke et al., 2011). In the class Flavobacteria, the family Cryomorphaceae contains many species that play a crucial role in the carbon and energy fluxes in marine environments (Kirchman, 2002). Therefore, this trend of decreasing Flavobacteria might be due to the decreasing possibility of receiving large tides.

\section{Conclusion}

In this study, higher resolution of soil microbial community structure in coastal saline-alkali soil under the T. chinensis woodland at Bohai Bay were revealed. The results showed that, except 19 known bacterial phyla, a large proportion of unclassified bacteria at the phylum level were found. The dominant bacterial structure was likely due to the arid environmental conditions of the coastal saline-alkali soil. The existing of $T$. chinensis is also another important factor to change the soil microbial community, via changing the niches for the microorganisms in coastal saline-alkali soil. The third impact factor could be the distance to the seashore line, which could affect certain groups of soil bacteria in this saline-alkali soil.

\section{Acknowledgements}

This study was funded by the National Natural Science Foundation of China (31470544, 41271265), a special financial grant from the China Postdoctoral Science Foundation (2013T60900), and the Science and Technology Projects in Gansu Province (1304NKCA135). 


\section{References}

Bull A T, Asenjo J A. 2013. Microbiology of hyper-arid environments: recent insights from the Atacama Desert, Chile. Antonie Van Leeuwenhoek, 103(6): 1173-1179.

Cao D, Shi F, Ruan W, et al. 2011. Seasonal changes in and relationship between soil microbial and microfaunal communities in a Tamarix chinensis community in the Yellow River Delta. African Journal of Biotechnology, 10(80): 18425-18432.

Carpentier A, Como S, Dupuy C, et al. 2013. Feeding ecology of Liza spp. in a tidal flat: Evidence of the importance of primary production (biofilm) and associated meiofauna. Journal of Sea Research, 92: 86-91.

Chanal A, Chapon V, Benzerara K, et al. 2006. The desert of Tataouine: an extreme environment that hosts a wide diversity of microorganisms and radiotolerant bacteria. Environmental Microbiology, 8(3): 514-525.

Chen M, Zhu J W, Shen J D, et al. 2008. The effect of Tamarix spp canopy on the soil enzyme activities and the microbial quantity. Acta Agriculturae Boreali-occidentalis Sinica, 17(2): 212-217. (in Chinese)

Cole J R, Chai B, Farris R J, et al. 2007. The ribosomal database project (RDP-II): introducing myRDP space and quality controlled public data. Nucleic Acids Research, 35: D169-D172.

Cottrell M T, Kirchman D L. 2000. Natural assemblages of marine proteobacteria and members of the Cytophaga-Flavobacter cluster consuming low-and high-molecular-weight dissolved organic matter. Applied and Environmental Microbiology, 66(4): 1692-1697.

Dhar P P, Al-Qarawi A A, Mridha M A U. 2015. Arbuscular mycorrhizal fungal association in Asteraceae plants growing in the arid lands of Saudi Arabia. Journal of Arid Land, 7(5): 676-686.

Dong Y M, Zhao C C, Cai Y, et al. 2013. Optimization of reed-specific degrading bacteria by response surfaces for remediation of crude oil-polluted soil in Xinjiang, China. Journal of Arid Land, 5(3): 408-414.

Doroshenko E A, Zenova G M, Zvyagintsev D G, et al. 2005. Spore germination and mycelial growth of streptomycetes at different humidity levels. Microbiology, 74(6): 690-694.

Finkel O M, Burch A Y, Elad T, et al. 2012. Distance-decay relationships partially determine diversity patterns of phyllosphere bacteria on Tamarix trees across the Sonoran Desert. Applied and Environmental Microbiology, 78(17): 6187-6193.

Gaskin J F, Schaal B A. 2002. Hybrid Tamarix widespread in US invasion and undetected in native Asian range. Proceedings of the National Academy of Sciences of the Unites States of America, 99(17): 11256-11259.

Graue J, Engelen B, Cypionka H. 2011. Degradation of cyanobacterial biomass in anoxic tidal-flat sediments: a microcosm study of metabolic processes and community changes. The ISME Journal, 6(3): 660-669.

Gray J P, Herwig R P. 1996. Phylogenetic analysis of the bacterial communities in marine sediments. Applied and Environmental Microbiology, 62(11): 4049-4059.

Hou X Y, Wu T, Yu L J, et al. 2012. Characteristics of multi-temporal scale variation of vegetation coverage in the circum Bohai Bay region, 1999-2009. Acta Ecologica Sinica, 32(6): 297-304. (in Chinese)

Jiang Z M, Chen Y X, Bao Y. 2012. Population genetic structure of Tamarix chinensis in the Yellow River Delta, China. Plant Systematics and Evolution, 298(1): 147-153.

Kim B S, Kim B K, Lee J H, et al. 2008. Rapid phylogenetic dissection of prokaryotic community structure in tidal flat using pyrosequencing. The Journal of Microbiology, 46(4): 357-363.

Kim B S, Oh H M, Kang H, et al. 2004. Remarkable bacterial diversity in the tidal flat sediment as revealed by $16 \mathrm{~S}$ rDNA analysis. Journal of Microbiology and Biotechnology, 14(1): 205-211.

Kim S J, Park S J, Cha I T, et al. 2014. Metabolic versatility of toluene-degrading, iron-reducing bacteria in tidal flat sediment, characterized by stable isotope probing-based metagenomic analysis. Environmental Microbiology, 16(1): 189-204.

Kirchman D L. 2002. The ecology of Cytophaga-Flavobacteria in aquatic environments. FEMS Microbiology Ecology, 39(2): 91-100.

Köpke B, Wilms R, Engelen B, et al. 2005. Microbial diversity in coastal subsurface sediments: a cultivation approach using various electron acceptors and substrate gradients. Applied and Environmental Microbiology, 71(12): 7819-7830.

Ksouri R, Falleh H, Megdiche W, et al. 2009. Antioxidant and antimicrobial activities of the edible medicinal halophyte Tamarix gallica L. and related polyphenolic constituents. Food and Chemical Toxicology, 47(8): 2083-2091.

Kuske C R, Ticknor L O, Miller M E, et al. 2002. Comparison of soil bacterial communities in rhizospheres of three plant species and the interspaces in an arid grassland. Applied and Environmental Microbiology, 68(4):1854-1863.

Lefahal M, Benahmed M, Louaar S, et al. 2010. Antimicrobial activity of Tamarix gallica L. extracts and isolated flavonoids. Advances in Natural and Applied Sciences, 4(3): 289-292.

Llobet-Brossa E, Rosselló-Mora R, Amann R. 1998. Microbial community composition of Wadden Sea sediments as revealed by fluorescence in situ hybridization. Applied and Environmental Microbiology, 64(7): 2691-2696.

Ma X M, Yin L K, Chen L. 2008. Study on vertical distribution of microorganismsin rhizosphere of populuseuphratica and Tamarix sp. in the lower reaches of the Tarim River, Xinjiang. Arid Zone Research, 25(2): 183-189. (in Chinese) 
Ma X M, Yin L K. 2009. The seasonal changes of plants' rhizosphere and non-rhizosphere microorganism in the desert riparian forest. Journal of Arid Land Resources and Environment, 23(5): 165-171. (in Chinese)

Margulies M, Egholm M, Altman W E, et al. 2005. Genome sequencing in open microfabricated high-density picoliter reactors. Nature, 437: 376-380.

McKenna P, Hoffmann C, Minkah N, et al. 2008. The macaque gut microbiome in health, lentiviral infection, and chronic enterocolitis. PLoS Pathogens, 8(4): e20.

Naether A, Foesel B U, Naegele V, et al. 2012. Environmental factors affect acidobacterial communities below the subgroup level in grassland and forest soils. Applied and Environmental Microbiology, 78(20): 7398-7406.

Nagy M L, Pérez A, Garcia-Pichel F. 2005. The prokaryotic diversity of biological soil crusts in the Sonoran Desert (Organ Pipe Cactus National Monument, AZ). FEMS Microbiology Ecology, 54(2): 233-245.

Orlando J, Alfaro M, Bravo L, et al. 2010. Bacterial diversity and occurrence of ammonia-oxidizing bacteria in the Atacama Desert soil during a "desert bloom" event. Soil Biology and Biochemistry, 42(7): 1183-1188.

Pruesse E, Quast C, Knittel K, et al. 2007. SILVA: a comprehensive online resource for quality checked and aligned ribosomal RNA sequence data compatible with ARB. Nucleic Acids Research, 35(21): 7188-7196.

Qvit-Raz N, Finkel O M, Al-Deeb T M, et al. 2012. Biogeographical diversity of leaf-associated microbial communities from salt-secreting Tamarix trees of the Dead Sea region. Research in Microbiology, 163(2): 142-150.

Roesch L F, Fulthorpe R R, Riva A, et al. 2007. Pyrosequencing enumerates and contrasts soil microbial diversity. The ISME Journal, 1: 283-290.

Saïdana D, Mahjoub M A, Boussaada O, et al. 2008. Chemical composition and antimicrobial activity of volatile compounds of Tamarix boveana (Tamaricaceae). Microbiological Research, 163(4): 445-455.

Saul-Tcherkas V, Steinberger Y. 2011. Soil microbial diversity in the vicinity of a Negev Desert shrub-Reaumuria negevensis. Microbial Ecology, 61(1): 64-81.

Schloss P D, Gevers D, Westcott S L. 2011. Reducing the effects of PCR amplification and sequencing artifacts on 16S rRNA-based studies. PLoS One, 6(12): e27310.

Sultanova N, Makhmoor T, Abilov Z A, et al. 2001. Antioxidant and antimicrobial activities of Tamarix ramosissima. Journal of Ethnopharmacology, 78(2-3): 201-205.

Szabolcs I. 1992. Salinization of soil and water and its relation to desertification. Desertification Control Bulletin, 21: 27-32.

Thomas D S G, Middleton N J. 1993. Salinization: new perspectives on a major desertification issue. Journal of Arid Environments, 24(1): 95-105.

Urakawa H, Kita-Tsukamoto K, Ohwada K. 1999. Microbial diversity in marine sediments from Sagami Bay and Tokyo Bay, Japan, as determined by 16S rRNA gene analysis. Microbiology, 145(11): 3305-3315.

Uroz S, Buée M, Murat C, et al. 2010. Pyrosequencing reveals a contrasted bacterial diversity between oak rhizosphere and surrounding soil. Environmental Microbiology Reports, 2(2): 281-288.

Wilms R, Köpke B, Sass H, et al. 2006a. Deep biosphere-related bacteria within the subsurface of tidal flat sediments. Environmental Microbiology, 8(4): 709-719.

Wilms R, Sass H, Köpke B, et al. 2006b. Specific bacterial, archaeal, and eukaryotic communities in tidal-flat sediments along a vertical profile of several meters. Applied and Environmental Microbiology, 72(4): 2756-2764.

Woyke T, Chertkov O, Lapidus A, et al. 2011. Complete genome sequence of the gliding freshwater bacterium Fluviicola taffensis type strain (RW262 ${ }^{\mathrm{T}}$ ). Standards in Genomic Sciences, 5(1): 21-29.

Yu Y, Lee C, Kim J, et al. 2005. Group-specific primer and probe sets to detect methanogenic communities using quantitative real-time polymerase chain reaction. Biotechnology and Bioengineering, 89(6): 670-679.

Zhang T, Shao M F, Ye L. 2011. 454 Pyrosequencing reveals bacterial diversity of activated sludge from 14 sewage treatment plants. The ISME Journal, 6(6): 1137-1147.

Zhang W, Zhang G S, Liu G X, et al. 2012. Bacterial diversity and distribution in the southeast edge of the Tengger Desert and their correlation with soil enzyme activities. Journal of Environmental Sciences, 24(11): 2004-2011.

Zhao X L, Zhou G S, Lv G H. 2009. The study on soil microbial characteristic under different types of vegetation in Liaohe Delta. Chinese Journal of Soil Science, 40(6): 1266-1269. (in Chinese)

Zhu G R, Xu X G, Ma Z W, et al. 2012. Spatial dynamics and zoning of coastal land-use change along Bohai Bay, China, during 1979-2008. Journal of Coastal Research, 28(5): 1186-1196.

Zhu H, Zhao C Y, Li J, et al. 2008. Distribution characteristics of soil MBC and relevant factors in woodland of Tamarix Ramasissima, central Asia. Acta Pedologica Sinica, 45(2): 375-379. (in Chinese) 